Military Technical College, Kobry El-Kobbah, Cairo, Egypt $9^{\text {th }}$ International Conference On Aerospace Sciences \& Aviation Technology

\title{
PERFORMANCE OF LOW EARTH ORBIT SATELLITE SYSTEMS WITH A GAUSSIAN MIXTURES TRAFFIC DISTRIBUTION
}

\author{
HEBATALLAH M. MOURAD* ABD EL-AZIZ M. EL-BASIONI** SHERIF S. EMAM ${ }^{\star \star \star *}$ \\ EMAD K. AL-HUSSAINI ${ }^{\star \star \star \star \star}$
}

\section{ABSTRACT}

A new traffic model for Low Earth Orbit (LEO) Satellite system is proposed. Two different cases are considered. The first case represents the situation in which the traffic load follows a bimodal contaminated Gaussian distribution. The second case considers the trimodal distribution. The parameters of this new distribution model are introduced and their effects on the Signal to Interference Ratio (SIR) and the capacity are investigated.

\section{KEYWORDS}

Low Earth Orbit Satellite Communication

\section{INTRODUCTION}

Low earth orbit satellite communication systems are one of the most appropriate systems to offer personal communications (PC) [1-3]. They can also provide additional advantages for the global communication networks, e.g., small propagation delay and loss, and high elevation angle in high latitude [4].

One of the most recent candidates for establishing the multiple access in LEO satellite systems is Code Division Multiple Access (CDMA). CDMA has higher capacity than TDMA and FDMA if voice activity and frequency reuse by spatial isolation are employed [5]. The non-uniform distribution of the traffic is a normal feature of our globe. However there are only few studies on the effect of this nonuniformity of the traffic on the performance of LEO systems. Performance analysis of

*Assistance professor, Dept. of Communication, Faulty of Engineering, Cairo University, Giza, Egypt.

** Lucent Technologies, Cairo Office.

*** PhD. Student.

**** Professor, Dept. of Communication, Faulty of Engineering, Cairo University, Giza, Egypt. 
LEO satellite communication systems with traffic non-uniformity was considered by Abbas, et al., [6]. In his analysis, the traffic model was assumed to have a Gaussian probability density function with variance $\omega^{2}$, which represents the traffic nonuniformity. The model represents the case of an isotated city. Due to the relative large area coverage of a LEO satellite, more than one inhabited area can exist in the coverage area of three consecutive satellites. In this case the Gaussian distribution will not be a good choice to represent the traffic non-uniformity. A (rather) more suitable distribution for a lot of practical cases is the Gaussian mixtures, which is considered in this paper.

This paper suggests a general traffic model that can resemble specific areas in the globe. It also discusses the effects of traffic non-uniformity on the performance of the LEO satellite communication system employing CDMA scheme. In section II we define the new traffic model. Section III contains the performance measure (SIR) for three adjacent satellites. Section IV contains the other performance measure namely the capacity for these three adjacent satellites. Section $V$ shows the numerical results. Section VI includes the conclusions

\section{TRAFFIC NON-UNIFORMITY IN LEO SATELLITE SYSTEMS}

In this section we cosider the distribution of the traffic in the area covered by the three satellites. In LEO satellite systems, the satellites are organized on a multiple orbit configuration. In the GlobalStar system for example the 48 satellites are organized in 8 orbits, each with 6 satellites

In this model shown in Fig. 1, an arc represents an area on the earth [7]. The figure shows the coverage and interference areas of each satellite. The coverage area is specified by the minimum elevation angle $\left(\theta_{\mathrm{min}}\right)$. The interference area of a satellite is specified by the final line of sight of that satellite. An area covered by two satellites will be denoted by "double coverage area". Bi represents the position of the ith satellite measured from the center of the earth

\section{- The Contaminated Gaussian Traffic Model}

To analyze the effect of traffic non-uniformity, we define the traffic distribution as:

$$
P(\alpha)=A \sum_{i=-M}^{M} \frac{\varepsilon_{i}}{\omega_{i}} \exp \left(\frac{-\left(\alpha-\mu_{i}\right)^{2}}{2 \omega_{i}^{2}}\right) \quad|\alpha|<\pi
$$

where $\alpha$ is the angular distance of any user from the origin measured by the angle at the center of the earth in radians, $\mu_{i}$ is the center of the $i^{\text {th }}$ populated area, $\omega_{i}$ is the nonuniformity parameter of the $i^{\text {th }}$ populated area and $\varepsilon_{i}$ is the weight of the $i^{\text {th }}$ populated area relative to the total traffic load. The number of populated areas will depend on " $M$ " and the values of $\varepsilon_{i}$ as will be shown later The constant $A$ is given by: 


$$
A=B \cdot\left[\int_{-3 \pi / N S}^{3 \pi / N} \sum_{i=-M}^{M} \frac{\varepsilon_{i}}{\omega_{i}} \exp \left[\frac{-\left(\alpha-\mu_{i}\right)^{2}}{2 \omega_{i}^{2}}\right] d \alpha\right]^{-1}
$$

where $B$ is the total traffic load for the three satellites between $-3 \pi / N_{s}<\alpha<3 \pi / N_{s}, N_{s}$, is the number of satellites in one orbit. For the sake of simplicity we will assume that $\omega_{i}=\omega$ for all i. Next, we shall consider two cases of interest, in particular, the cases of two and three populated areas respectively.

\section{- The Case Of Two Populated Areas}

In this case, we put in (1), $M=1$,

$$
\varepsilon_{-1}=\varepsilon, \quad \varepsilon_{0}=0, \quad \varepsilon_{1}=1-\varepsilon, \quad \mu_{1}=\mu_{1} \quad \mu_{-1}=-\mu
$$

Thus, we obtain the conventional contaminated Gaussian distribution given by:

$$
P(\alpha)=\frac{A}{\omega}\left[\varepsilon \exp \left[\frac{-(\alpha+\mu)^{2}}{2 \omega^{2}}\right]+(1-\varepsilon) \exp \left[\frac{-(\alpha-\mu)^{2}}{2 \omega^{2}}\right]\right]
$$

The probability density function (p.d.f.) of (3) is shown in Fig. 2.

\section{- The Case Of Three Populated Areas}

In this case $P(\alpha)$ is given by:

$$
P(\alpha)=\frac{A}{\omega}\left[\varepsilon_{1} \exp \left[\frac{-(\alpha-\mu)^{2}}{2 \omega^{2}}\right]+\varepsilon_{2} \exp \left[\frac{-\alpha^{2}}{2 \omega^{2}}\right]+\varepsilon_{3} \exp \left[\frac{-(\alpha+\mu)^{2}}{2 \omega^{2}}\right]\right]
$$

Which is obtained by substituting in (1), $\mu_{0}=0, \mu_{1}=\mu$ and $\mu_{-1}=-\mu . \varepsilon_{1}, \varepsilon_{2}$ and $\varepsilon_{3}$ are the weights of these populated areas and can take any arbitrary values satisfying that $\varepsilon_{1}+\varepsilon_{2}+\varepsilon_{3}=1$. The p.d.f. of (4) is depicted in Fig. 3 .

\section{EVALUATION OF SIGNAL TO INTERFERENCE RATIO (SIR)}

Consider three adjacent satellites. The $1^{\text {st }}$ and $3^{\text {rd }}$ satellites have $\beta_{1}, \beta_{3}$ equal to $-2 \pi$ $/ N_{s}, 2 \pi / N_{s}$ respectively, while $\beta_{2}=0$. The received power of satellite $i$ from any user within its service area is $S_{i}$. Furthermore equal service areas and perfect power control are assumed [6]. Any satellite will be affected by the interference comming from all users in the satellite interference area. Users are assumed to have omni directional antennas. So, the interference of the $1^{\text {st }}$ satellite is given by: 


$$
\begin{aligned}
& I_{1}=S_{1} \int_{-\infty \pi i N}^{-n / N} P(\alpha) d \alpha \\
& +S_{0} \int_{-2 \pi / N_{,}-\beta}^{-3=/ N_{i}} P(\alpha) \frac{\left[R+h-R \cos \left(4 \pi / N_{s}+\alpha\right)\right]^{2}+R^{2} \sin ^{2}\left(4 \pi / N_{t}+\alpha\right)}{\left[R+h-R \cos \left(2 \pi / N_{s}+\alpha\right)\right]^{2}+R^{2} \sin ^{2}\left(2 \pi / N_{t}+\alpha\right)} d \alpha \\
& +S_{2} \int_{-\infty / N}^{-2 \pi / N_{*}+\beta} P(\alpha) \frac{[R+h-R \cos (\alpha)]^{2}+R^{2} \sin ^{2}(\alpha)}{\left[R+h-R \cos \left(2 \pi / N_{s}+\alpha\right)\right]^{2}+R^{2} \sin ^{2}\left(2 \pi / N_{t}+\alpha\right)} d \alpha
\end{aligned}
$$

Similarly the interference of the $2^{\text {nd }}$ satellite can be written as follows:

$$
\begin{aligned}
I_{2} & =S_{2} \int_{-3 \pi / N_{s}}^{-\pi / N_{s}} P(\alpha) d \alpha \\
& +S_{1} \int_{-\beta_{l}}^{-\pi / N_{s}} P(\alpha) \frac{\left[R+h-R \cos \left(2 \pi / N_{s}+\alpha\right)\right]^{2}+R^{2} \sin ^{2}\left(2 \pi / N_{s}+\alpha\right)}{[R+h-R \cos (\alpha)]^{2}+R^{2} \sin ^{2}(\alpha)} d \alpha \\
& +S_{3} \int_{\pi / N_{s}}^{\beta_{l} P(\alpha)} \frac{\left[R+h-R \cos \left(2 \pi / N_{s}-\alpha\right)\right]^{2}+R^{2} \sin ^{2}\left(2 \pi / N_{s}-\alpha\right)}{[R+h-R \cos (\alpha)]^{2}+R^{2} \sin ^{2}(\alpha)} d \alpha
\end{aligned}
$$

Finally the interference of the $3^{\text {rd }}$ satellite is given by:

$$
\begin{aligned}
I_{3} & =S_{3} \int_{\pi / N_{s}}^{3 \pi / N_{s}} P(\alpha) d \alpha \\
& +S_{2} \int_{2 \pi / N_{s}-\beta_{I}}^{\pi i N_{s}} P(\alpha) \frac{[R+h-R \cos (\alpha)]^{2}+R^{2} \sin ^{2}(\alpha)}{\left[R+h-R \cos \left(2 \pi / N_{s}-\alpha\right)\right]^{2}+R^{2} \sin ^{2}\left(2 \pi / N_{s}-\alpha\right)} d \alpha \\
& +S_{4} \int_{3 \pi / N_{s}+\beta_{I}}^{2 \pi / N_{I}} \frac{\left[R+h-R \cos \left(4 \pi / N_{s}-\alpha\right)\right]^{2}+R^{2} \sin ^{2}\left(4 \pi / N_{s}-\alpha\right)}{\left[R+h-R \cos \left(2 \pi / N_{s}-\alpha\right)\right]^{2}+R^{2} \sin ^{2}\left(2 \pi / N_{s}-\alpha\right)} d \alpha
\end{aligned}
$$

Where the first term in (5), (6), and (7) is the interference from the same satellite by its own users and the second and third terms are from users of both adjacent satellites $[8-10]$. Finally the $i^{\text {th }}$ SIR is $\left(S_{i} / I_{i}\right)$.

\section{CAPACITY MEASUREMENT}


Another performance measure in LEO satellite is the system capacity. To find the equation for the capacity, we first determine the ratio of the bit energy-to-noise density for the $\mathrm{i}^{\text {th }}$ satellite [6]:

$$
\left(E_{b} / N_{0}\right)_{i}=\frac{B W / R_{b}}{\left(I_{i} / S_{i}\right)+\left(\eta / S_{i}\right)}
$$

Where the numerator is the ratio of the total bandwidth $(B W)$, to the information bit rate $\left(R_{b}\right)$, and the denominator is the total interference-to-signal ratio plus the ratio of background noise $(\eta)$, to signal. The thermal noise is assumed Gaussian with zero mean, and variance $(\eta) . / /$ is the total interference reaching the $i^{\text {th }}$ satellite, (for $i=1$, 2,3 ) as given by $(5),(6)$, and $(7)$, and is proportional to the total traffic load, $B$. Therefore the maximum amount of traffic that the system can support for a given condition can be denoted as:

$$
B_{i}=\frac{I_{i}}{\left[I_{i}\right]_{B=1}}
$$

Where $\left[I_{i}\right]_{B=1}$ means the interference, $I_{i}$ calculated at $B=1$. Solving (8) for $I_{i}$ and substituting it in (9) we have

$$
B_{i}=\left(\frac{B W / R_{b}}{\left(E_{b} / N_{0}\right)_{i}}-\frac{\eta}{S_{i}}\right) \cdot \frac{S_{i}}{\left[I_{i}\right]_{B=1}}
$$

From this equation, we can derive the maximum traffic $B_{i}$ (capacity) for a certain $E_{b} / N_{0}$ on the $i^{\text {th }}$ satellite for $a, R_{b}, \eta$ given $B W$, and $S_{i}$. Capacity of the satellites (SAT, $\mathrm{SAT}_{2}$ and $\mathrm{SAT}_{3}$ ) are calculated, and shown in Figures (8) to (11) for different parameter values.

\section{NUMERICAL RESULTS}

The SIR and the capacity are calculated for the three consecutive satellites SAT, $\mathrm{SAT}_{2}$ and $\mathrm{SAT}_{3}$ for the case of two populated areas and three populated areas assuming that all received satellites power are equal. In each case we study the effect of two parameters namely the mean and the weight of the $i^{\text {th }}$ populated area $(\mu$, $\varepsilon)$. For the sake of illustration it is important to mention that the service areas of SAT, $\mathrm{SAT}_{2}$ and $\mathrm{SAT}_{3}$ extends from (-0.86 rad to $\left.-0.29 \mathrm{rad}\right),(-0.29 \mathrm{rad}$ to $0.29 \mathrm{rad})$ and (0.29 rad to $0.86 \mathrm{rad}$ ) respectively.

The effect of the weighting parameter for the 1st case is shown in Fig.4. It is a plot of the SIR of the satellites for $\mu=\gamma / 2,\left(\gamma=2 \pi / N_{s}\right)$ and for two different values of $\varepsilon$. SAT and $\mathrm{SAT}_{3}$ have the same SIR for $\varepsilon=0.5$ since the distribution of the users is the same for both satellites which can be deduced from Fig. 2 where the number of users under $\mathrm{SAT}_{1}=\mathrm{SAT}_{3}=25$ while the number of users under $\mathrm{SAT}_{2}=50$. However, for $\varepsilon$ $=0.1$ there is large unbalance between $\mathrm{SAT}_{1}$ and $\mathrm{SAT}_{3}$ where the number of users 
under $\mathrm{SAT}_{1}=45$ while number of users under $\mathrm{SAT}_{3}=5$. Therefore $\mathrm{SAT}_{1}$ and $\mathrm{SAT}_{2}$ can be considered as a Dense Traffic Satellite (DTS) while SAT 3 as Sparse Traffic Satellites (STS). Furthermore, the SIR for the $2^{\text {nd }}$ satel ite changes slightly with the variation of $\varepsilon$ because the number of users in its service area is almost the same $(\sim$ $50)$.

The effect of varying $\mu_{1}$ for $\varepsilon=0.5$ is shown in Fig. 5 . As $\mu$ approaches 0 , the traffic model approaches that of one populated area under the satellite SAT 2 . Thus the discrepancy in SIR between the satellites increases, then SAT $T_{2}$ is DTS and SAT 1 and $\mathrm{SAT}_{3}$ are STS's.

The performance in case of three populated areas is shown in Figures (6) and (7). Fig. 6 shows the SIR of SAT SAT $_{2}$ and $\mathrm{SAT}_{3}$ for $\varepsilon_{1}=\varepsilon_{3}=0.1$ and $\varepsilon_{2}=0.8$, as $\mathrm{\mu}$ changes from $y / 3$ to $2 y / 3$. For such a low weight of $\varepsilon_{1}$ and $\varepsilon_{3}$, we will have SAT 1 and $\mathrm{SAT}_{3}$ as STS's and SAT 2 as DTS. Fig. 7 shows the effect of $\mu$ for $\varepsilon_{1}=\varepsilon_{3}=0.4$ and $\varepsilon_{2}$ $=0.2$. It is clear that the effect of $\mu$ is more pronounced for these values of $\varepsilon$. With the relatively higher values of $\mu$, the three satellites become more balanced (i.e, the discrepancy between DTS, STS is smaller). The unbalance increases as $\mu$ decreases.

Notice that results of the case of Gaussian distribution traffic [2] is included in our analysis for $\mu=0$.

Figures (8) to (11) present the effect of $\mu_{i}, \varepsilon_{i}$ on the capacity of the system. In these Figures, $\mathrm{BW}=1.25 \mathrm{MHz}, \mathrm{Rb}=8 \mathrm{~Kb} / \mathrm{s}, \mathrm{S} i \eta \bar{l}=-1 \mathrm{~dB}$, and $\mathrm{Eb} / \mathrm{NO}$ is greater than $5 \mathrm{~dB}$ Since the capacity is proportional to the SIR when $B=1$, the results of the capacity confirm those of the SIR.

\section{CONCLUSIONS}

A Gaussian mixture traffic model was introduced, it was shown that this distribution can fit specific cases of the globe, by the proper choice of the parameters $\varepsilon_{i}, \mu_{i}$ and $\omega_{i}$ for all i. Previous models are considered as special cases of this suggested model. We can conclude that varying $\varepsilon, \mu$, controls mainly the number of users in each service area thus affects the performance of each satellite. The three adjacent satellites will be balanced if this number is the same for all of these satellites. Otherwise there will be an unbalance between the three satellites resulting in having STS, and DTS. It was shown that the SIR increases from 7 to $16 \mathrm{~dB}$ depending on the chosen distribution parameters.

\section{REFERENCES}

[1] D. Chakraborty, "Survivable communication concept via multiple low earthorbiting satellites, "IEEE Trans. On AES. Vol. 25, no. 6, pp. 879-889,1989

[2] J.Kaniyil, J.Takei,S. Shimamoto, Y. Onozato, T. Usui, I. Oka, T. Kawabata, "A global message network employing low earth-orbiting satellites, "IEEE Jour. On SAC, vol. 10, no. 2, pp. 418-428, 1992.

[3] R. J. Leopold, "The Iridium communication system, " in Proc. Of the ICCS/ISITA. Singapore, pp. 451-455, 1992 
[4] M. Katayama, A. Ogawa, and N. Morinage," Satellite communication systems with low earth orbits and the effect of Doppler shift, ${ }^{n}$ IEICE Trans., vol. J67B-II, no. 5, 1993, PP. 382-390

[5] K. S. Gilhousen, I. M. Jacobs, R. Padovani, A. J. Viterbi, L. A. Weaver, Jr.,andC. E. Wheatly III, "On the capacity of a cellular CDMA system, " IEEE Trans. Veh. Tech., vol. 40, no. 2, pp. 303-312, 1991

[6] A. Jamalipour, M. Katayama, T. Yamazato, and A. Ogawa,"a performance analysis on the effects traffic nonuniformity in low earth-orbit satellite communication systems," in Proc. 16th Symp. Inform. Theory. Applicat. (SITA'93) (Japan), Vol. 1, 1993, PP. 203-206.

[7] A. Jamalipour et al., "performance of an integrated voice/Data system in nonuniform traffic low earth orbit satellite communication systems," IEEEJ. Select. Areas Commun., Vol. 13, No. 2, 1995, PP. 465-473.

[8] Pritchard, W. L., H. G. Suyderhoud, and R. A. Nelson, Satellite Communication Engineering, 2nd ed, Englewood Cliffs, NJ: Prentice- Hall, 1987.

[9] Roddy, D., Satellite Communications, Englewood Cliffs, NJ: PrenticeHall, 1989.

[10] Werner, M., et al., "Analysis of System Parameters for LEO/ICO-Satellite Communication Networks," IEEE J. Select. Areas Commun., Vol. 13, No. 2, 1995, pp.371-381. 


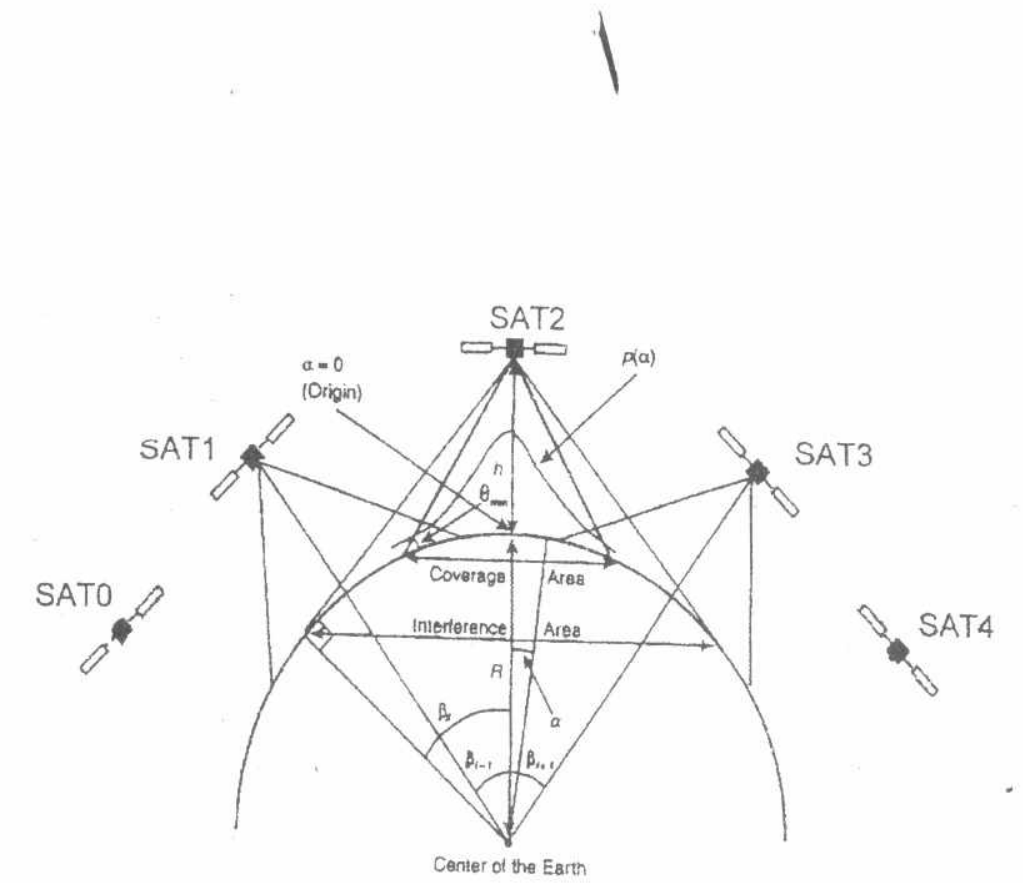

Fig.1. The System model 
Proceedings of the $9^{\text {th }}$ ASAT Conference, 8-10 May 2001

Paper AV-08

757

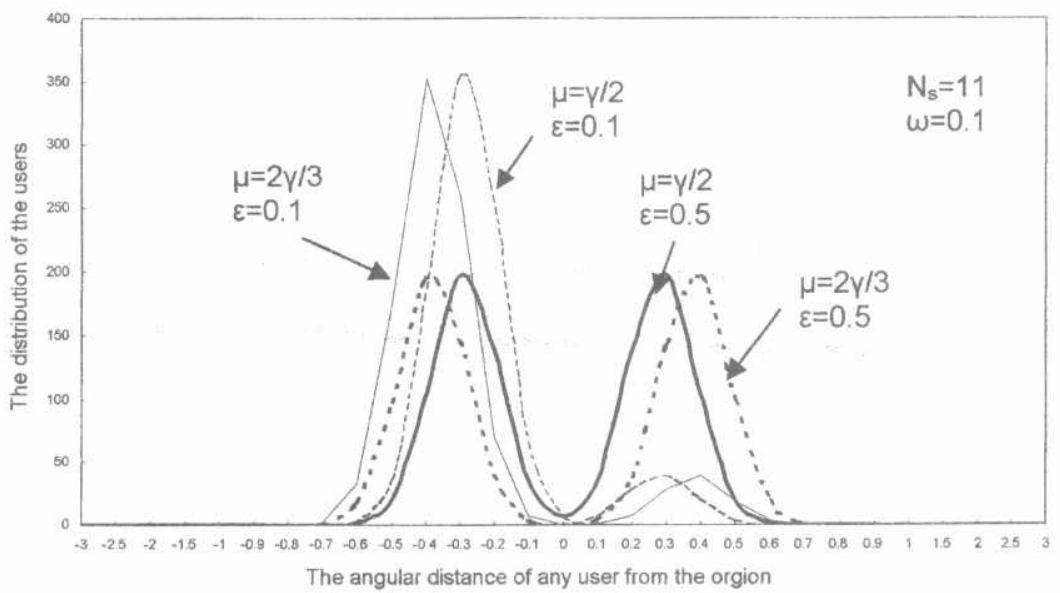

Fig.2. The distribution of the users in case of two populated areas 


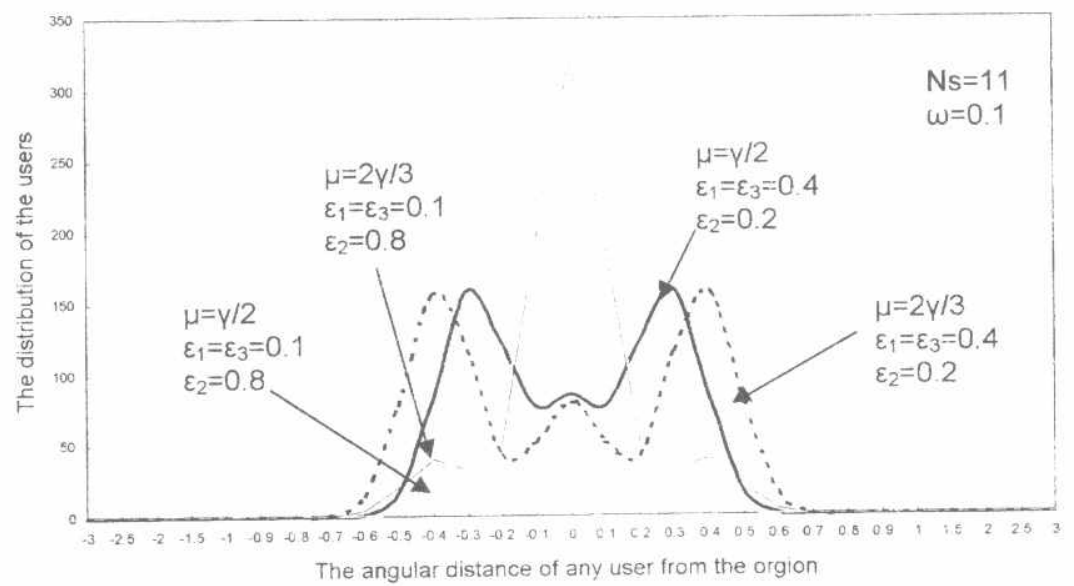

Fig.3. The distribution of the users in case of three populated areas 
Proceedings of the $9^{\text {th }}$ ASAT Conference, 8-10 May 2001

Paper AV-08

759

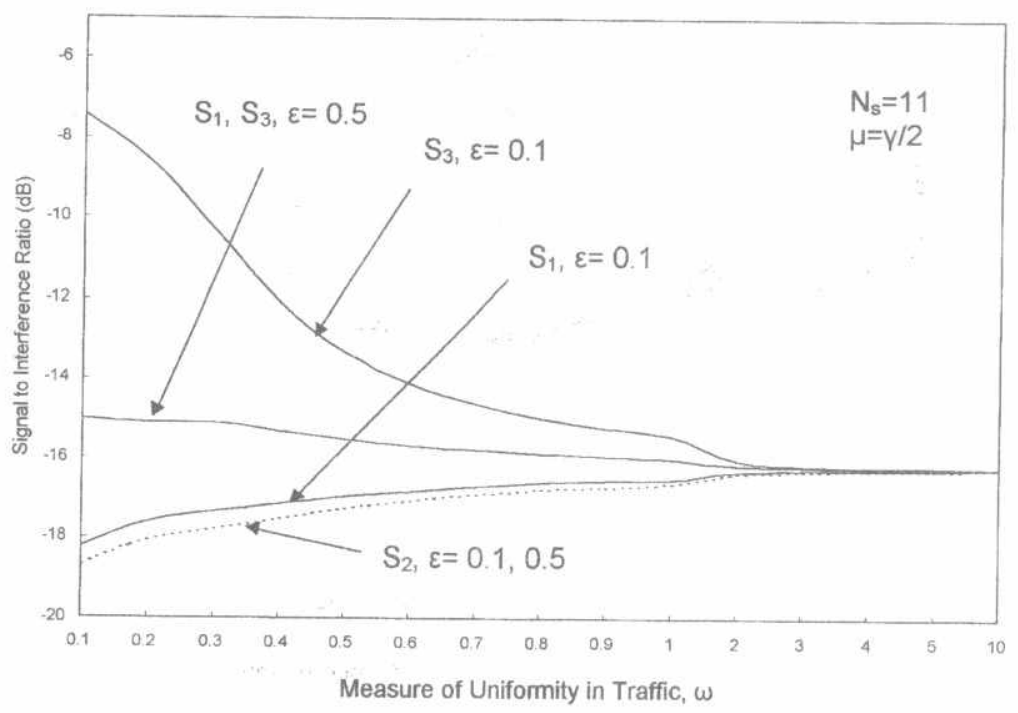

Fig.4. SIR in case of two populated areas as a function of traffic nonuniformity for $\mu=$ $\gamma / 2, \varepsilon=0.1,0.5$ 


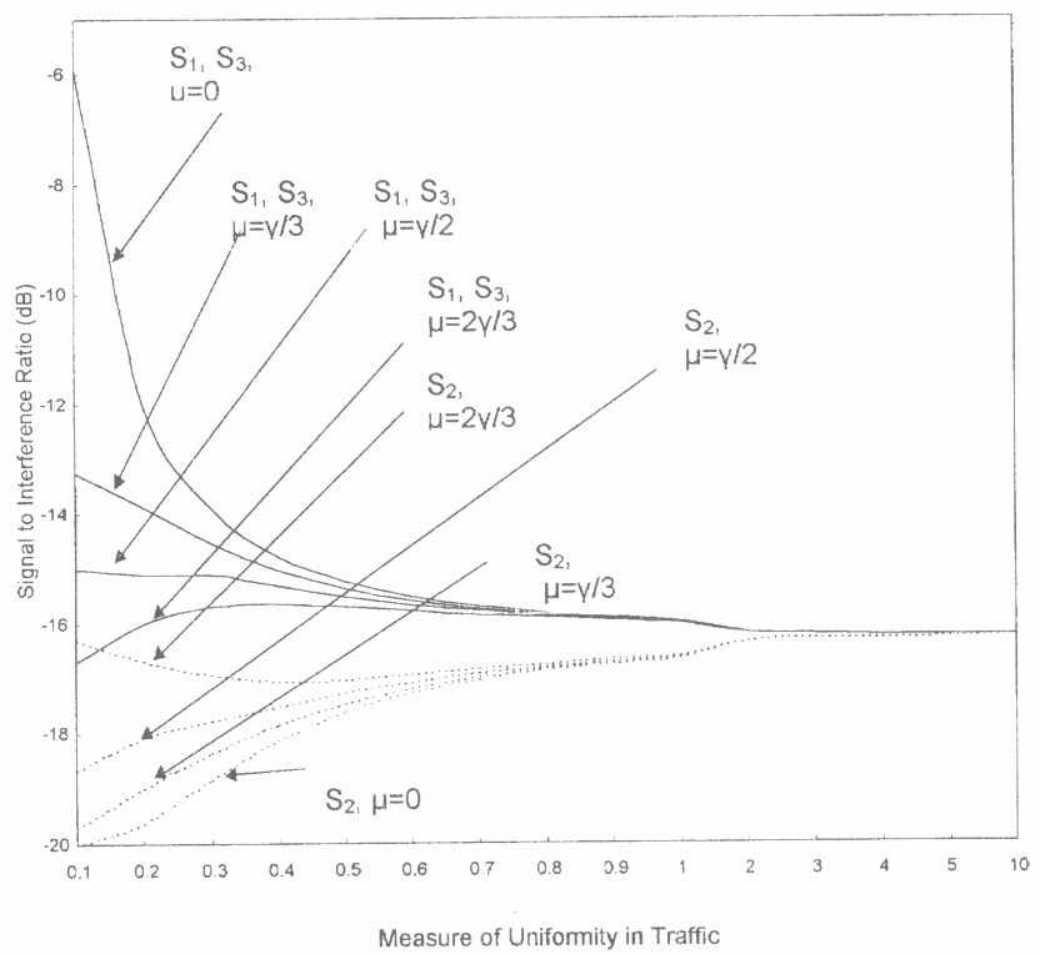

Fig. 5. SIR in case of two populated areas as a function of traffic nonuniformity for $\varepsilon=$ 0.5 , different values of $\mu$ 


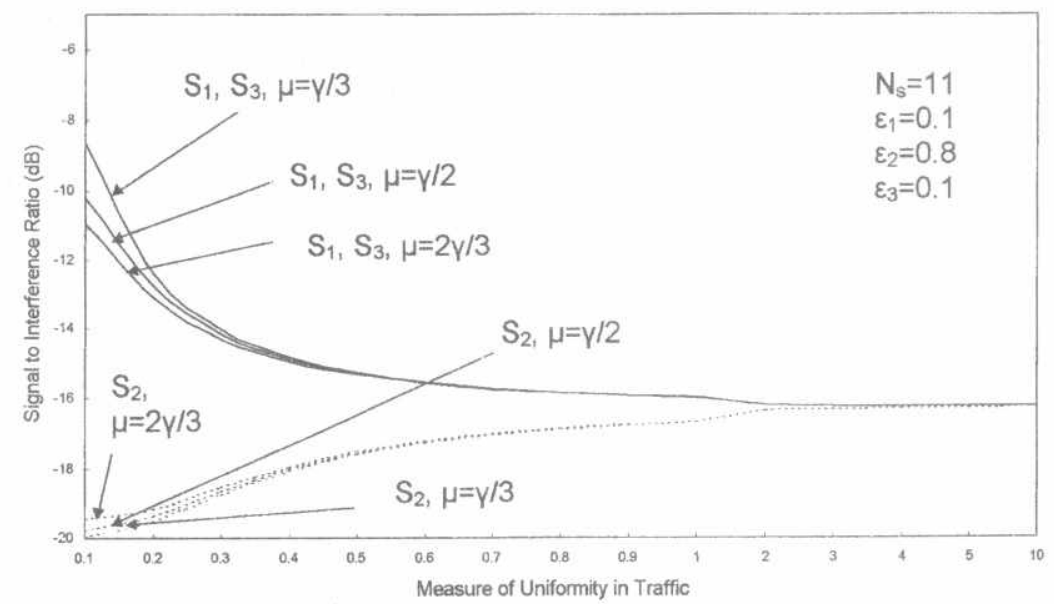

Fig.6. SIR in case of three populated areas as a function of traffic nonuniformity for different values of $\mu$ 


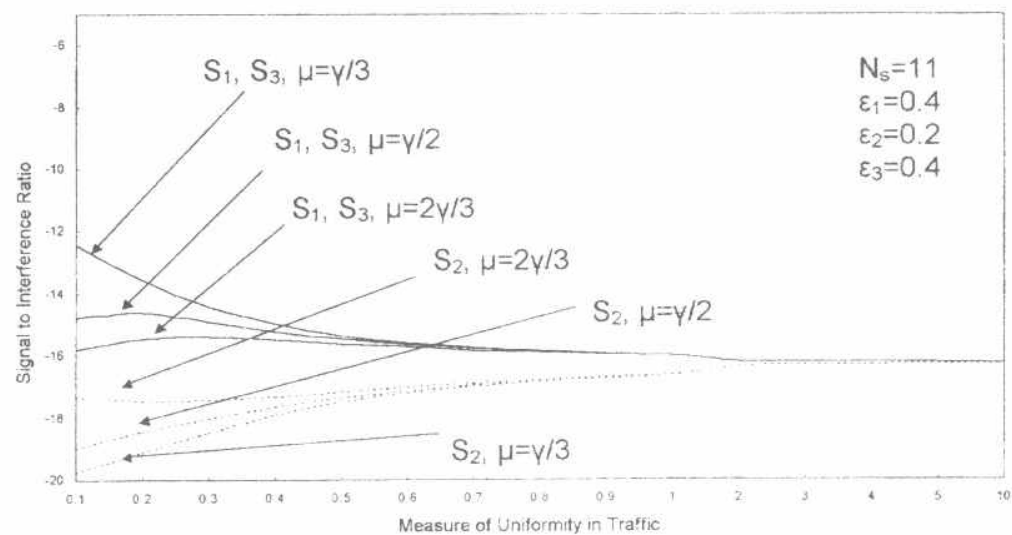

Fig.7. SIR in case of three populated areas as a function of traffic nonuniformity for different values of $\mu$ 


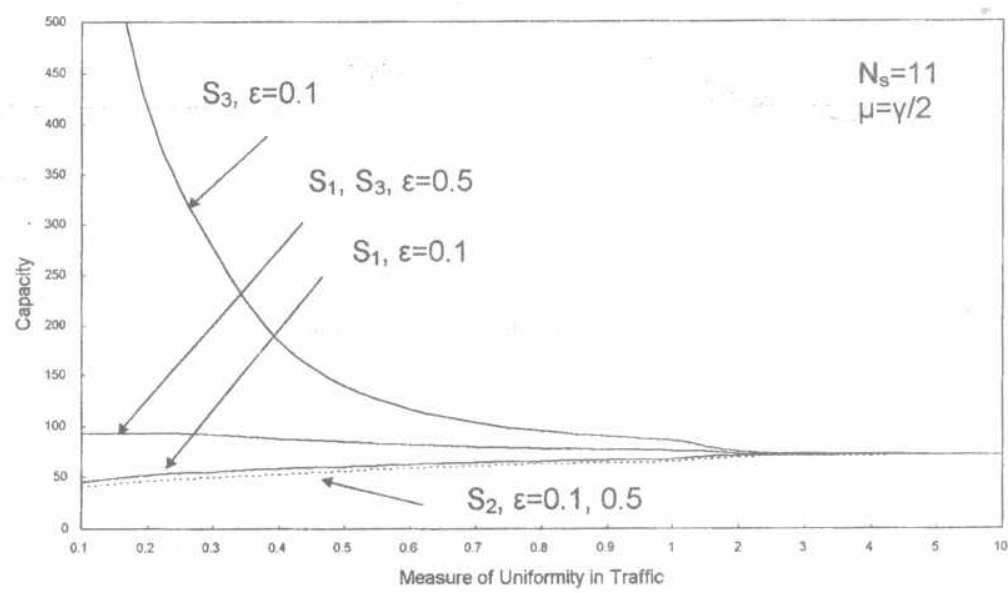

Fig.8. Capacity in case of two populated areas as a function of traffic nonuniformity for $\mu=\gamma / 2, \varepsilon=0.1,0.5$ 


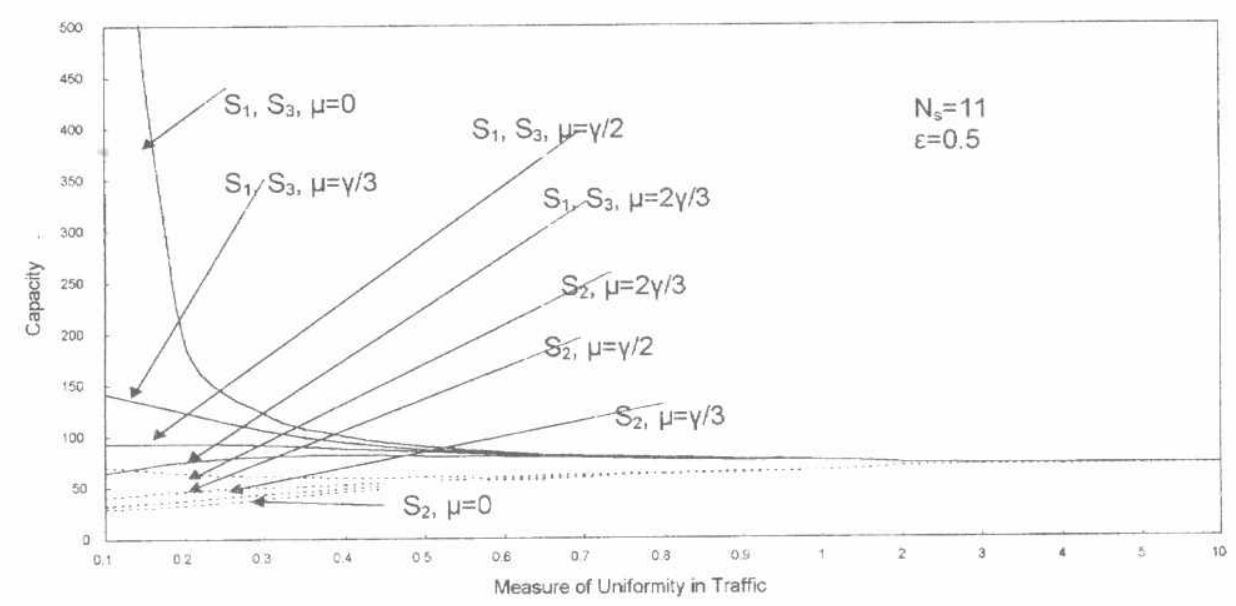

Fig.9. Capacity in case of two populated areas as a function of traffic nonuniformity for $\varepsilon=0.5$, different values of $\mu$ 


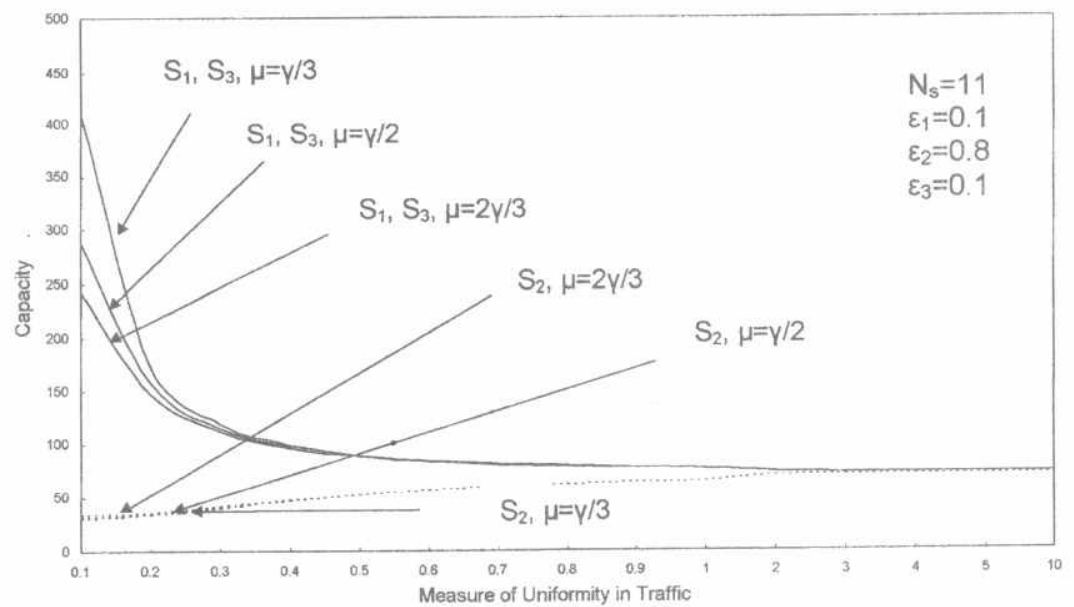

Fig. 10. Capacity for three populated areas as a function of traffic nonuniformity for different values of $\mu$ 
Proceedings of the $9^{\text {th }}$ ASAT Conference, 8-10 May 2001

Paper AV -08

766

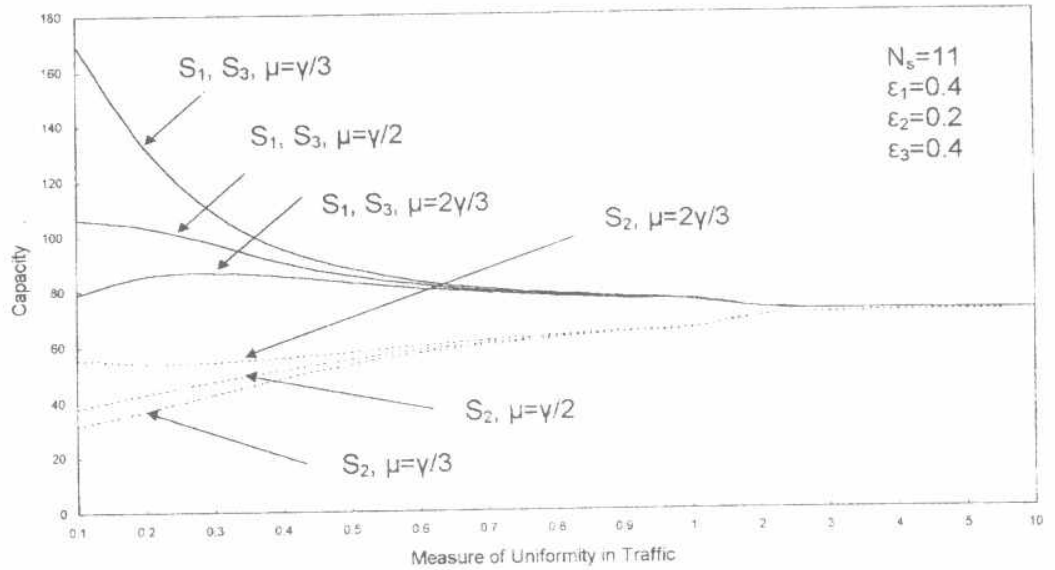

Fig.11. Capacity for three populated areas as a function of traffic nonuniformity for different values of $\mu$ 